\title{
Human MutY homolog induces apoptosis in etoposide-treated HEK293 cells
}

\author{
SOO-HYUN HAHM ${ }^{1}$, JI HYUNG CHUNG ${ }^{2}$, LIA AGUSTINA ${ }^{1}$, SE-HEE HAN ${ }^{1}$, IN-SOO YOON ${ }^{1}$, \\ JONG-HWA PARK ${ }^{3}$, LIN-WOO KANG $^{1}$, JIN WOO PARK $^{4}$, JONG JOO NA $^{4}$ and YE SUN HAN ${ }^{1}$ \\ ${ }^{1}$ Department of Advanced Technology Fusion, Konkuk University, Gwangjin-gu, Seoul 143-701; \\ ${ }^{2}$ Yonsei Integrative Research Institute for Cerebral \& Cardiovascular Diseases (YIRIC), Yonsei University Health System, \\ Seoul 120-752; ${ }^{3}$ Department of Genetic Engineering and Graduate School of Biotechnology, Kyung Hee University, \\ Yongin 446-701; ${ }^{4}$ BioActs, DKC Corporation, Gojan-dong, Namdong-gu, Incheon 405-820, Republic of Korea
}

Received July 10, 2012; Accepted August 31, 2012

DOI: $10.3892 / \mathrm{ol} .2012 .921$

\begin{abstract}
Etoposide (ETP) treatment of ataxia telangiectasia mutated (ATM) and Rad3-related protein (ATR)-, topoisomerase-binding protein-1 (TopBP1) and human MutY homolog (hMYH)-depleted cells results in a significant reduction in apoptotic signaling. The association between ATR or TopBP1 and hMYH increased following ETP treatment. In hMYH knockdown cells, the interaction between ATR and TopBP1 decreased following ETP treatment. We suggest that hMYH functions as a sensor of ETP-induced apoptosis. The results suggest that in the absence of hMYH, cells are unable to recognize the damage signal and the ATR pathway is not activated.
\end{abstract}

\section{Introduction}

DNA damage response is essential for the maintenance of genome integrity (1-5). As a complex, the DNA damage response involves the recognition of DNA damage, activation of DNA damage-responsive protein kinases, signal amplification by downstream protein kinases and activation of the effector proteins that trigger various cellular processes. At low DNA damage levels, activation of the DNA damage response results in cell cycle arrest and DNA repair. However, at higher levels or under severe conditions, DNA damage response signaling frequently results in cell death by apoptosis (1-5).

Phosphoinositide 3-kinase-related protein kinases, ataxia telangiectasia mutated proteins (ATM) and Rad3-related

Correspondence to: Dr Ye Sun Han, Department of Advanced Technology Fusion, Konkuk University, 1 Hwayang-dong, Gwangjin-gu, Seoul 143-701, Republic of Korea

E-mail: yshan@konkuk.ac.kr

Key words: human MutY homolog, ataxia telangiectasia mutated, Rad3-related protein, topoisomerase-binding protein-1, apoptosis, etoposide proteins (ATR), are the key regulators of the DNA damage response (1-8). Once activated, ATM and ATR regulate an array of substrates, including Chk1 and Chk2, which culminate in DNA repair, cell cycle arrest and/or apoptosis. In the canonical model, ATR activation involves the recruitment of the ATR-ATR interacting protein (ATR-ATRIP) and Rad9-Hus1-Rad1 (9-1-1) protein complexes to the DNA damage site via replication protein A (RPA). As a result, the 9-1-1 complex brings topoisomerase-binding protein-1 (TopBP1) (ATR activator) close to ATR for ATR activation $(1,4,8)$. Mammalian TopBP1 functions at the DNA replication checkpoint $(9,10)$ and has multiple BRCA1 C-terminal (BRCT) repeats, which usually function in tandem to bind phosphoproteins $(11,12)$. TopBP1 colocalizes with ATR-ATRIP at the sites of DNA replication stress $(9,10)$. The N-terminus of TopBP1 is required for its recruitment and the resulting activation of ATR via interaction with Rad9 in mammalian cells (13).

Human MutY homolog (hMYH) is a base excision repair DNA glycosylase that excises misincorporated adenine opposite 7,8-dihydro-8-oxoguanine (8-oxoG), a product of oxidative DNA damage. Furthermore, in hMYH-disrupted cells, the phosphorylation of ATR and Chk1 is decreased by hydroxyurea (HU) or ultraviolet (UV) treatment (14). The hMYH is known to interact with 9-1-1 (15), and a recent study revealed that it also interacts with ATR and MutS $\alpha$ via the human MutS homolog (hMSH) 6 subunit $(14,16)$.

The mismatch repair protein $\mathrm{hMSH} 2$ may interact with ATR and participate in ATR activation during DNA damage, leading to apoptosis. Therefore, hMSH2-deficient cells are more resistant to apoptosis (17). The mismatch repair and MYH repair pathways share many common features. First, both pathways function immediately following DNA replication to distinguish newly synthesized DNA strands from their parental counterparts $(18,19)$. Second, hMYH and hMSH6 interact with the replication proteins proliferating cell nuclear antigen and RPA and colocalize at the same replication foci $(20,21)$. Finally, both pathways are involved in mutation avoidance following DNA oxidation. Therefore, we suggest that the interaction between hMYH and hATR is the same as hMSH 2 and hATR. 
Etoposide (ETP), a topoisomerase II inhibitor, is known to induce apoptosis and activate ATR via TopBP1 $(22,23)$; however, it is unclear how hMYH is involved during the ETP response. In this study, MYH, ATR and TopBP1 knockdown cells were treated with ETP. In the absence of these proteins, the cells were more resistant to ETP-induced apoptosis. We determined for the first time that hMYH interacts with hTopBP1 or hATR in HEK293 cells, and that this interaction is increased following ETP treatment. However, when hMYH is disrupted, the interaction between hATR and hTopBP1 is decreased following ETP treatment. Since hATR is inactive, the apoptosis signal cannot transduce to the downstream proteins, specifically p-Chk2 and p-p53.

\section{Materials and methods}

Cell lines. Human embryonic kidney HEK293 cells were grown in Dulbecco's modified Eagle's medium (Invitrogen Life Technologies, Carlsbad, CA, USA) supplemented with $10 \%$ fetal bovine serum (Invitrogen Life Technologies) and $1 \%$ penicillin-streptomycin solution (Sigma, St. Louis, MO, USA) at $37^{\circ} \mathrm{C}$ in a $5 \% \mathrm{CO}_{2}$ incubator. Prior to the experiments, HEK 293 cells were seeded into 6-well plates at a density of $1 \times 10^{6}$ cells per well and incubated for $24 \mathrm{~h}$.

siRNA construction and transfection into cells. The optimum siRNA sequences for the knockdown of endogenous hMYH were designed and purchased from the Stealth ${ }^{\mathrm{TM}}$ RNAi program of Invitrogen Life Technologies. siRNA corresponding to nucleotides 415-439 of the green fluorescence protein (GFP) was used as a negative control. The siMYH and siGFP sequences and transfection method were conducted as previously described (14). ATR siRNA (sc-29763) and TopBP1 siRNA were purchased from Santa Cruz Biotechnology, Inc. (sc-41068; Santa Cruz, CA, USA). ATR and TopBP1 knockdown were conducted according to the manufacturer's instructions.

Protein extraction and western blot analysis. HEK293 cells were harvested, washed with phosphate-buffered saline (PBS) and lysed with lysis buffer containing $50 \mathrm{mM}$ Tris- $\mathrm{HCl}$ phenylmethanesulfonylfluoride, protease and a phosphatase inhibitor cocktail (Sigma), for $1 \mathrm{~h}$ at $4^{\circ} \mathrm{C}$ with occasional vortexing. Protein extracts were collected following centrifugation at $16,000 \mathrm{x} \mathrm{g}$ for $20 \mathrm{~min}$, and protein concentration was determined using a Bio-Rad DC protein assay kit (Bio-Rad, Hercules, CA, USA). Protein extracts that were resolved on 8 or $12 \%$ sodium dodecyl sulfate (SDS)-polyacrylamide gels were transferred onto PVDF membranes (GE Healthcare Worldwide, Princeton, NJ, USA). The membranes were blocked with $5 \%$ non-fat dried milk in Tris-buffered saline with $0.05 \%$ Tween-20 and then incubated with antibodies against ATR, Chk1, Chk2, phospho-Chk1 (Ser-345), phospho-Chk2 (Thr-68), $\beta$-actin (all from Santa Cruz Biotechnology, Inc.), phospho-ATR (Ser-428), caspase 9, caspase 7, p-p53 (Ser-15; all from Cell Signaling Technology, Inc., Beverly, MA, USA), TopBP1 (Abcam, Cambridge, UK) and hMYH (Abnova, Teipei, Taiwan). Membranes were then incubated with horseradish peroxidase-conjugated secondary antibodies (Santa Cruz Biotechnology, Inc.). Protein bands were detected using enhanced chemiluminescence (ECL) Pico western blotting detection reagents (Pierce Biotechnology, Inc., Rockford, IL, USA).

Immunofluorescence microscopy. To determine the subcellular location of ATR, TopBP1 and hMYH, HEK293 cells were seeded onto polylysine-coated coverslips and treated with $25 \mu \mathrm{M}$ ETP for $48 \mathrm{~h}$. At room temperature, cells were fixed with $4 \%$ paraformaldehyde in PBS for $30 \mathrm{~min}$ and permeabilized with $0.25 \%$ Triton X-100 in PBS for 30 min. After blocking with $1 \%$ bovine serum albumin in PBS containing $0.5 \%$ Tween-20 (PBS-T) for $30 \mathrm{~min}$, cells were incubated with ATR (1:100; Santa Cruz Biotechnology, Inc.), TopBP1 (1:500; Abcam) and MYH monoclonal antibodies (1:100; Abnova) for $2 \mathrm{~h}$. The cells were washed 3 times for $15 \mathrm{~min}$ each in PBS and incubated with Alexa 488-conjugated anti-mouse IgG (1:100; Sigma), fluorescein isothiocyanate (FITC)-conjugated anti-rabbit IgG (1:100; Sigma) for $2 \mathrm{~h}$. Cells were rinsed 3 times with $1 \mathrm{ml}$ PBS and analyzed using a confocal fluorescence microscope (Olympus FV-1000; software, Olympus Fluoview; Olympus, Center Valley, PA, USA).

Co-immunoprecipitation. Co-immunoprecipitation (IP) of endogenous proteins using an ATR antibody was conducted using the ImmunoCruz ${ }^{\mathrm{TM}}$ IP/WB Optima B System (Santa Cruz Biotechnology, Inc.) according to the manufacturer's instructions. IP was conducted using rabbit anti-ATR, and immunoblot (IB) analysis was conducted using rabbit anti-ATR, rabbit anti-TopBP1 and mouse anti-MYH. To determine the effect of ETP on MYH-ATR and MYH-TopBP1 interaction, a similar co-IP procedure was conducted using mouse anti-MYH for co-IP, and IB analysis using rabbit anti-ATR, rabbit anti-TopBP1 and mouse anti-MYH.

Statistical analysis. Experiments were conducted in triplicate and statistical analyses were conducted using the Student's $\mathrm{t}$-test. Data are expressed as the mean, and $\mathrm{P}<0.05$ was considered to indicate a statistically significant difference.

\section{Results}

Effect of ETP on HEK293 cell proliferation. We examined the effect of ETP on cell viability by treating cells with 3 different concentrations of ETP $(10,25$ and $50 \mu \mathrm{M})$ for various periods of time. After 24,48 and $72 \mathrm{~h}$ of treatment, cell viability was determined using the 3-(4,5-dimethylthiazol-2-yl)-2,5-diphenyltetrazolium bromide (MTT) assay. Fig. 1A shows that treatment with ETP greatly reduced cell viability compared with the control cells. ETP induced apoptosis in HEK293 cells in a time- and dose-dependent manner. We determined that treatment with $25 \mu \mathrm{M}$ ETP was the best condition for our subsequent experiments.

To assess the molecular mechanism of ETP-induced apoptosis, we examined the expression of the $85-\mathrm{kDa}$ cleaved form of poly-ADP ribose polymerase (PARP), an apoptosis marker, and hMYH, an apoptosis-related protein (25). Cells were treated with $25 \mu \mathrm{M}$ ETP for 24,48 or $72 \mathrm{~h}$. As shown in Fig. 1B, cleavage of PARP was observed after 48 and $72 \mathrm{~h}$. The hMYH protein level increased in a time-dependent manner, indicating the involvement of hMYH in apoptosis. Taken 
A

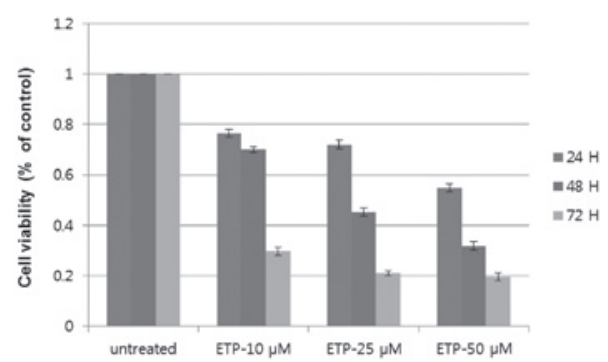

B

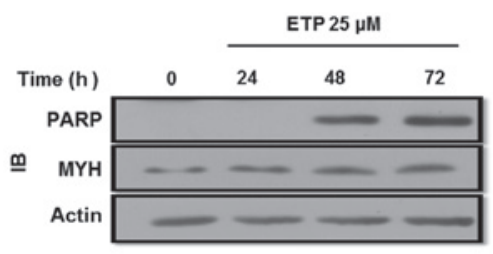

C

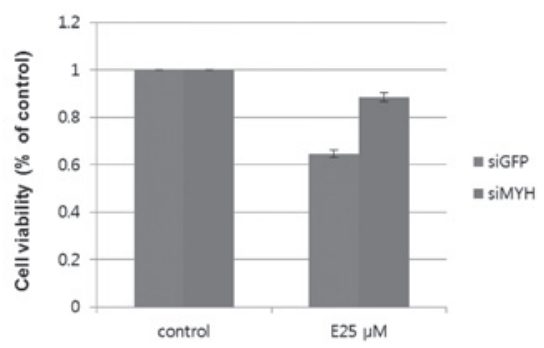

Figure 1. ETP-induced apoptosis in HEK293 cells. (A) ETP treatment reduced cell viability. Cells were treated with 0, 10, 25 and 50 $\mu$ M ETP. After 24 , 48 and $72 \mathrm{~h}, 20 \mu \mathrm{l}$ MTT was added to each well and incubated for an additional $4 \mathrm{~h}$. The results shown are the mean \pm SD of 3 independent experiments. (B) ETP-induced cleavage of PARP following $48 \mathrm{~h}$ of incubation. Cells were incubated with $25 \mu \mathrm{M}$ ETP for 24,48 and $72 \mathrm{~h}$. Cell lysates were subjected to SDS-PAGE. PARP and MYH expression levels were determined by western blotting using the indicated antibodies. (C) hMYH knockdown reduced cell viability following ETP treatment. Cells were transfected with siGFP or siMYH and incubated for $24 \mathrm{~h}$. Cells were then treated with $25 \mu \mathrm{M}$ ETP for $48 \mathrm{~h}$. Cell viability was determined by the MTT assay. The results shown are the mean \pm SD of 3 independent experiments. ETP, etoposide; IB, immunoblot; PARP, poly-ADP ribose polymerase; MYH, MutY homolog; GFP, green fluorescence protein. MTT, 3-(4,5-dimethylthiazol-2-yl)-2,5-diphenyltetrazolium bromide; SDS-PAGE, sodium dodecyl sulfate polyacrylamide gel electrophoresis; hMYH, human MYH.

A

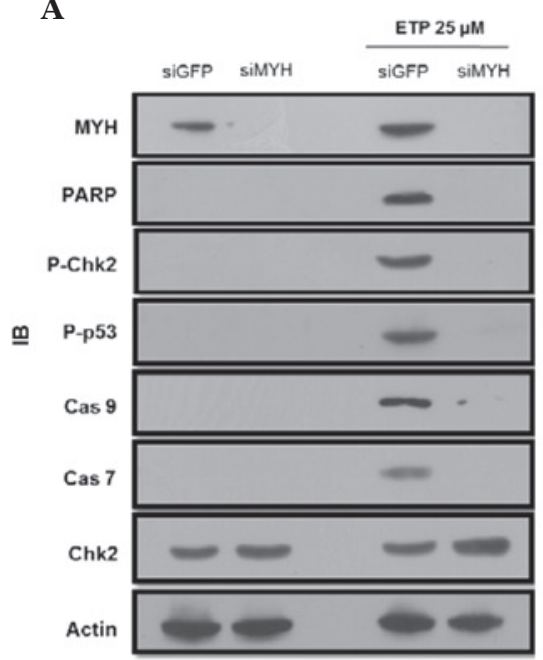

B

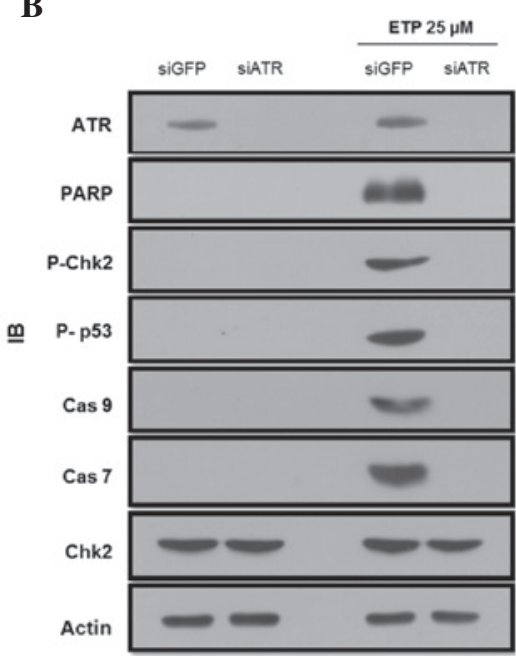

C

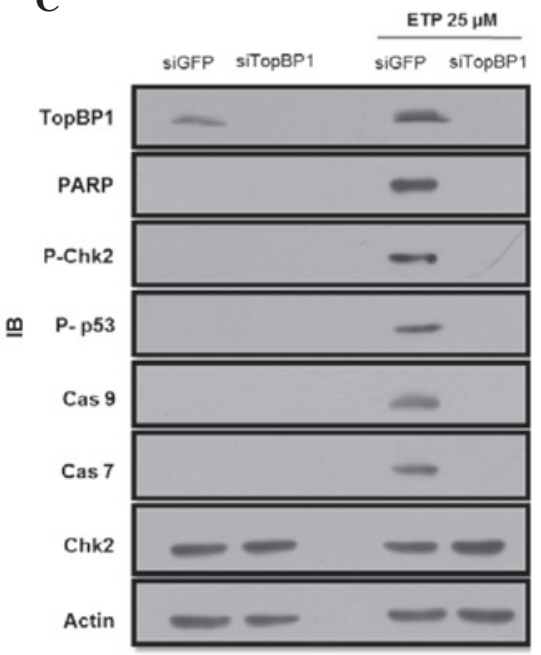

Figure 2. MYH, ATR and TopBP1 knockdown decreases ETP-induced apoptosis in HEK293 cells. (A) MYH knockdown reduces the activation of apoptosis-related proteins. Control or siMYH transfected cells were treated with $25 \mu \mathrm{M}$ ETP for $48 \mathrm{~h}$. Total cell lysates were used for IB analysis of p-Chk2 (threonine-68), p-p53 (threonine-15), caspase 9, caspase 7 and MYH. $\beta$-actin was used as a loading control. (B and C) The same experiment as in A, however, the cells were transfected with siRNA for either ATR or TopBP1. IB, immunoblot; MYH, MutY homolog; PARP, poly-ADP ribose polymerase; Cas, caspase; GFP, green fluorescence protein; ETP, etoposide; ATR, Rad3-related protein; TopBP1, topoisomerase-binding protein-1.

together, treatment with $25 \mu \mathrm{M}$ ETP for $48 \mathrm{~h}$ was the best condition for the subsequent experiment.

To further determine the effect of hMYH on cell viability, cells transfected with MYH-siRNA or control-siRNA were treated with $25 \mu \mathrm{M}$ ETP for $48 \mathrm{~h}$. Fig. 1C shows that cell viability, as determined by the MTT assay, decreased by $\sim 40 \%$ in the control cells, while only a $17 \%$ decrease was observed in MYH-siRNA cells. These results suggested the involvement of hMYH in apoptotic signaling during ETP treatment.
hMYH, hTopBPl and hATR deficient cells are resistant to ETP-induced apoptosis and DNA-damage signaling. Recently, it has been reported that ATR phosphorylation upon HU or UV treatment was decreased in hMYH-disrupted HEK293 and HaCaT cells (14). ATR is known to phosphorylate Chk1 and Chk2 (14,15), and here, we observed a phosphorylation of Chk2 only in control-transfected cells, but not in siMYH cells following ETP treatment (Fig. 2A). This result suggests that hMYH is required for ATR-mediated Chk2 activation under these conditions. The phosphorylation of Chk1 observed was similar to 


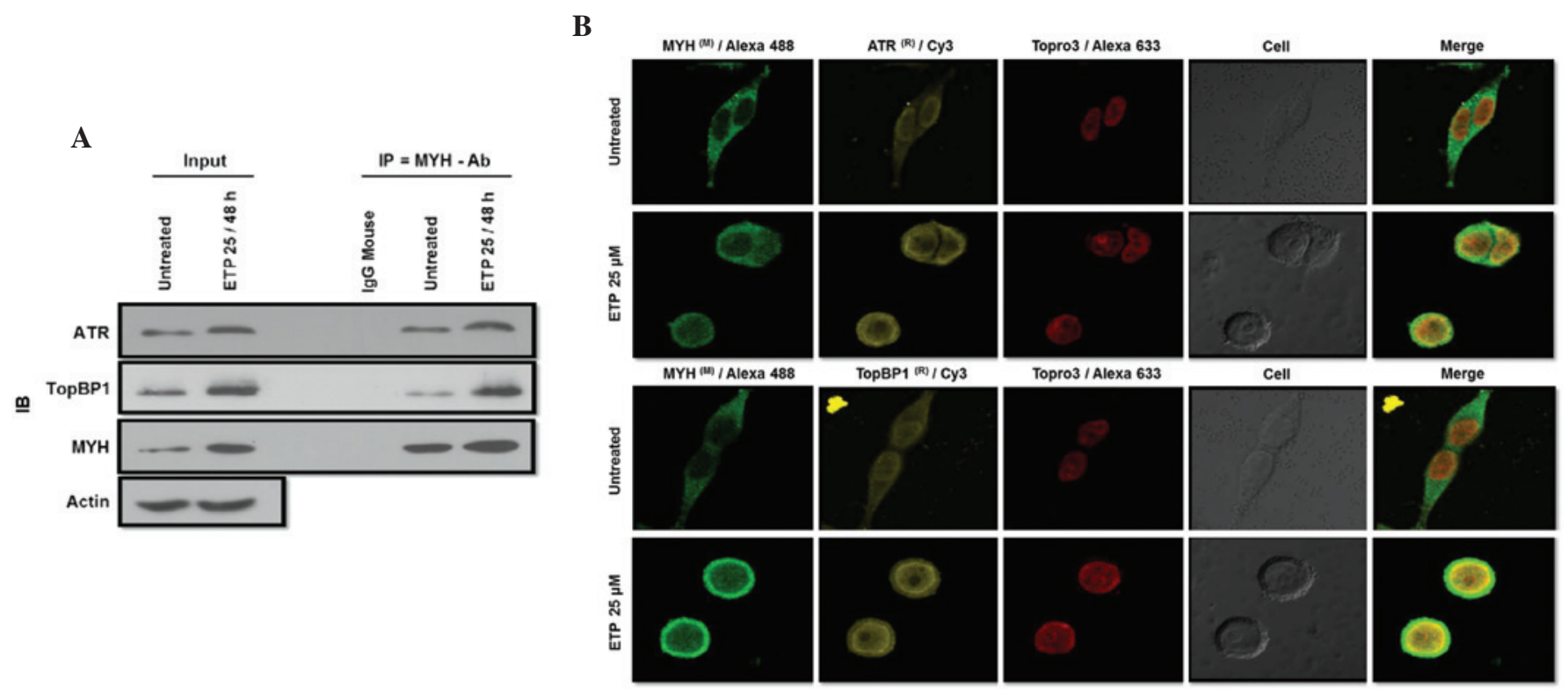

Figure 3. Interaction of MYH with ATR or TopBP1 increased following ETP treatment. (A) ATR or TopBP1 interact with MYH increases following treatment with ETP. Cells were treated with $25 \mu \mathrm{M}$ ETP for $48 \mathrm{~h}$. Cells were lysed and total cell lysates were used for co-IP with MYH antibody. IP samples were analyzed by western blotting with ATR or TopBP1 and MYH antibodies. $\beta$-actin was used as a loading control. (B) ATR or TopBP1 and MYH co-localized following treatment with ETP. Cells were cultured overnight on coverglass bottom dishes, then treated with $25 \mu \mathrm{M}$ ETP for $48 \mathrm{~h}$, and fixed with $4 \%$ paraformaldehyde and permeabilized with $0.1 \%$ Triton X-100 in PBS. Cells were then stained with antibodies against ATR or TopBP1 (FITC; yellow), MYH (Alexa $\left.{ }^{\circledR} 488 / G r e e n\right)$ and To-pro ${ }^{\circledR}$ (Red). IB, immunoblot; ATR, Rad3-related protein; TopBP1, topoisomerase-binding protein-1; MYH, MutY homolog; IP, immunoprecipitation; Ab, antibody. ETP, etoposide; PBS, phosphate-buffered saline; FITC, fluorescein isothiocyanate.

that of Chk2 (data not shown). Since transactivation of proapoptotic proteins through the p53-dependent signaling pathway is an important apoptotic mechanism, we focused on Chk2 because of its involvement in p53 regulation and ETP-induced apoptosis $(22,24)$. As shown in Fig. 2A, ETP-induced p53 phosphorylation was abrogated in siMYH-transfected cells.

Caspase is a well-known key molecule in DNA damage-induced apoptosis (22). The activation of caspase 9 and caspase 7 was examined via western blot analysis in hMYH knockdown and control cells. ETP treatment induced the cleavage of inactive 47-kDa procaspase 9 into smaller, detectable, active $37-\mathrm{kDa}$ fragments, and the cleavage of inactive $32-\mathrm{kDa}$ procaspase 7 into the $20-\mathrm{kDa}$ active form in control cells (Fig. 2A). However, the active forms of caspase 9 and 7 were not detected in hMYH knockdown cells following ETP treatment.

To determine the role of ATR relative to Chk2 or p53 in ETP-induced apoptosis, cells were treated with either control or ATR siRNA for $24 \mathrm{~h}$, and then treated with ETP for $48 \mathrm{~h}$. We observed increased phosphorylation or activation of Chk2 and p53 in control siRNA-transfected cells (Fig. 2B). However, the active forms of caspase 9 and 7 were not observed in hATR knockdown cells.

Since hTopBP1 is known to activate hATR, we observed the apoptosis pattern in hTopBP1 knockdown cells. Fig. 2C shows that apoptosis was decreased in hTopBP1 knockdown cells in comparison to the control cells, and similar results were observed in hMYH and hATR knockdown cells.

hMYH associates with ATR or TopBP1 in HEK293 cells during ETP treatment. ETP treatment activated hATR, hTopBP1 and hMYH (A-T-M), which triggered a signaling cascade leading to apoptosis. However, it is unclear how A-T-M is activated and regulates the apoptosis pathway. To determine whether hMYH regulates hATR or hTopBP1 in DNA damage models, we conducted a co-IP assay to confirm that hMYH interacts with hATR or hTopBP1. Cells were treated with $25 \mu \mathrm{M}$ ETP for $48 \mathrm{~h}$, and whole cell extracts were used. As shown in Fig. 3A, ETP treatment induced the association of hMYH-hATR and hMYH-hTopBP1. Our results suggest that hMYH interacts with hATR or hTopBP1 and participates in A-T-M activation during ETP treatment, which leads to the DNA damage response and signals apoptosis.

A critical function of hATR or hTopBP1 activation during genotoxic stress is the accumulation of hATR or hTopBP1 in the nucleus, where signaling proteins form multiple nuclei and interact in response to DNA damage $(6,11)$. To examine the changes in hATR and hTopBP1 localization, we conducted immunofluorescence (IF) experiments. We treated cells with $25 \mu \mathrm{M}$ ETP for $48 \mathrm{~h}$, and conducted IF assays to determine whether hMYH and hATR or hTopBP1 occupied the same subcellular locations during genotoxic stress. In untreated control cells, light hMYH staining was observed in the cytoplasm (Fig. 3B). However, following ETP treatment, we observed a higher intensity hMYH staining that translocated to the nucleus, indicating an increased expression. Staining of hATR and hTopBP1, which are known to localize to the nucleus, also increased in intensity following ETP treatment. Superimposition of hMYH-dependent green fluorescence with hATR or hTopBP1-dependent yellow fluorescence resulted in yellow-green images in cells. Topro3 Red (red fluorescence) is a nuclear marker. These results are consistent with the interaction of hMYH with hTopBP1 or hATR and their colocalization in cells. 


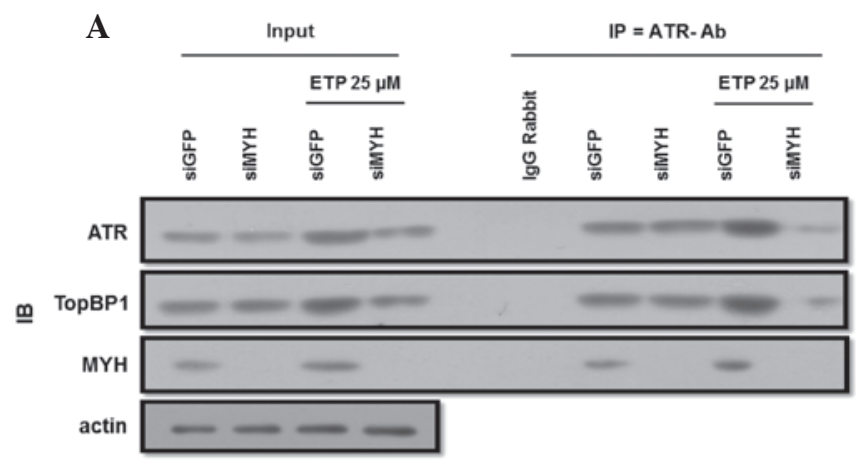

B

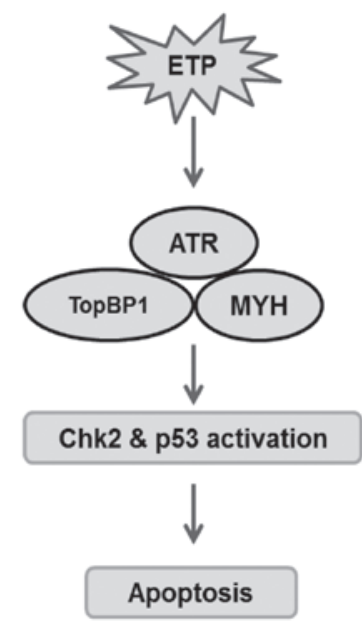

Figure 4. Knockdown of hMYH abrogated the interaction between ATR and TopBP1. (A) Co-IP and western blot analysis were conducted using lysates of cells transfected with siGFP or siMYH for $24 \mathrm{~h}$. Cells were treated with $25 \mu \mathrm{M}$ ETP for $48 \mathrm{~h}$ and co-IP was conducted using an ATR antibody. IP samples were further analyzed by western blotting with ATR, MYH and TopBP1 antibodies. Rabbit IgG was used as a control for co-IP. (B) A model of hMYH-mediated ATR activation in DNA damage signaling. DNA damage signaling involving ATR pathway activation is initiated by $\mathrm{hMYH}$ For ATR activation, hMYH interacts with ATR and TopBP1 at damaged sites, leading to the activation of Chk2 and p53, culminating in cell cycle arrest and apoptosis. IB, immunoblotting; ATR, Rad3-related protein TopBP1, topoisomerase-binding protein-1; hMYH, human MutY homolog; ETP, etoposide; GFP, green fluorescence protein; IP, immunoprecipitation; Ab, antibody.

ATR and TopBPl interaction decreased in hMYH-deficient cells during ETP treatment. The decrease in hMYH expression results in decreased apoptotic signaling following ETP treatment (Fig. 2A). Furthermore, hMYH interacts with hATR or hTopBP1 and this interaction increased following treatment with ETP (Fig. 3A). To observe the implication of hATR, hTopBP1 and hMYH interaction in this pathway, we conducted co-IP assays using ATR antibody. Cells were transfected with siGFP, as a control, or siMYH. Then, cells were treated with or without ETP. Total cell lysates were used in co-IP, and the presence of A-T-M was observed using western blot analysis.

A decreased hMYH expression was observed in MYH knockdown cells (Fig. 4A), while changes in ATR and TopBP1 expression levels were not observed in MYH knockdown cells. Following treatment with ETP, the expression of A-T-M increased. Furthermore, the co-IP result suggests that the interaction between hATR and hTopBP1 was decreased in MYH knockdown cells following treatment with ETP.

\section{Discussion}

hMYH interacts not only with hATR, but also with the human MutS homologs (hMSH2/hMSH6) via the hMSH6 subunit $(14,16)$. hMSH2 directly participates in ATR recruitment and activation, leading to DNA damage signaling and subsequent apoptosis. Therefore, hMSH2 deficiency increases the resistance of cells to apoptosis (17). Another study suggests that the nuclear isoforms of hMYH initiate cell death by sensing adenine opposite 8-oxoG during nuclear DNA replication, thus suppressing tumorigenesis. In addition, accumulation of 8-oxoG in mitochondrial DNA and initiation cell death by MYH may also contribute to the tumor suppression (25).

In this study, we implicated the DNA glycosylase, hMYH, in ETP-induced apoptosis, and revealed that hMYH knockdown cells reduce Chk2 (T-68) and p53 (T-15) phosphorylation. We also observed similar results in ATR and TopBP1 knockdown cells (Fig. 2). This result suggests the possibility that hATR, hTopBP1 and hMYH (A-T-M) function in the same pathway. This was accompanied by the suppression of proapoptotic protein expression and decreased apoptosis. The interaction between hMYH with ATR and TopBP1 is dependent on ETP treatment (Fig. 3).

In hMYH knockdown cells, ATR and TopBP1 interaction decreases following ETP treatment (Fig. 4A). We suggest that hMYH functions as a sensor in ETP induced apoptosis. In the absence of hMYH, cells cannot recognize the damage signal, and thus the ATR pathway is not activated, which results in tumor development $(14,25)$.

Based on these observations, we suggest new pathways for A-T-M sensor activation (Fig. 4B). This pathway is initiated by $\mathrm{MYH}$, which recruits and activates ATR-related proteins in ETP-induced apoptosis. In summary, binding of MYH directly participates in ATR and TopBP1 activation in DNA damage signaling, leading to apoptosis. An MYH protein deficiency increases the resistance of cells to apoptosis.

\section{Acknowledgements}

This study was supported by the project from the Advanced Technology Center (ATC) Support Program of the Ministry of Knowledge Economy (Republic of Korea; Grant No. 10033024), the Basic Science Research Program through the National Research Foundation of Korea (NRF; Grant No. 2012R1A1A3005889) and the World Class University (WCU; Grant No. R33-2008-000-1071) program through the Korea Science and Engineering Foundation funded by the Ministry of Education, Science and Technology.

\section{References}

1. Harper JW and Elledge SJ: The DNA damage response: ten years after. Mol Cell 28: 739-745, 2007.

2. Barket J, Bartkova J and Lukas J: DNA damage signaling guards against activated oncogenes and tumor progression. Oncogene 26: 7773-7779, 2007.

3. Zhou BB and Elledge SJ: The DNA damage response: putting checkpoint in perspective. Nature 408: 433-439, 2000.

4. Jackson SP and Bartek J: The DNA-damage response in human biology and disease. Nature 461: 1071-1078, 2009.

5. Sancar A, Lindsey-Boltz LA, Unsal-Kaçmaz K and Linn S: Molecular mechanisms of mammalian DNA repair and the DNA damage checkpoints. Annu Rev Biochem 73: 39-85, 2004. 
6. Abraham RT: PI 3-kinase related kinases: 'big' players in stress-induced signaling pathways. DNA repair 3: 883-887, 2004.

7. Hurley PJ and Bunz F: ATM and ATR: components of an integrated circuit. Cell cycle 6: 414-417, 2007.

8. Cimprich KA and Cortez D: ATR: an essential regulator of genome integrity. Nat Rev Mol Cell Biol 9: 616-628, 2008.

9. Mäkiniemi M, Hillukkala T, Tuusa J, Reini K, Vaara M, Huang D, Pospiech H, Majuri I, Westerling T, Mäkelä TP and Syväoja JE: BRCT domain-containing protein TopBP1 Functions in DNA replication and damage response. J Biol Chem 276: 30399-30406, 2001.

10. Garcia V, Furuya $\mathrm{K}$ and Carr AM: Identification and functional analysis of TopBP1 and its homologs. DNA Repair 4: 1227-1239, 2005.

11. Manke IA, Lowery DM, Nguyen A and Yaffe MB: BRCT repeats as phosphopeptide-binding modules involved in protein targeting. Science 302: 636-639, 2003.

12. Yu X, Chini CC, He M, Mer G and Chen J: The BRCT domain is a phosphor-protein binding domain. Science 302: 639-642, 2003.

13. Delacroix S, Wagner JM, Kobayashi M, Yamamoto $K$ and Karnitz LM: The Rad9-Hus1-Rad1 (9-1-1) clamp activates checkpoints signaling via TopBP1. Genes Dev 21: 1472-1477, 2007.

14. Hahm SH, Park JH, Ko SI, Lee YR, Chung IS, Chung JH, Kang LW and Han YS: Knock-down of human MutY homolog (hMYH) decreases phosphorylation of checkpoint kinase 1 (Chk1) induced by hydroxyurea and UV treatment. BMB Rep 44: 352-357, 2011.

15. Luncsford PJ, Chang DY, Shi G, Bernstein K, Madabushi A, Patterson DN, Lu AL and Toth EA: A structural hinge in eukaryotic MutY homologues mediates catalytic activity and Rad9-Rad1-Hus1 checkpoint complex interactions. J Mol Biol 403: 351-370, 2010

16. Gu Y, Parker A, Wilson TM, Bai H, Chang DY and Lu AL: Human MutY homolog, a DNA glycosylase involved in base excision repair, physically and functionally interacts with mismatch repair proteins human MutS homolog 2/Human MutS homolog 6. J Biol Chem 277: 11135-11142, 2002.
17. Pabla N, Ma Z, Mcllhatton MA, Fishel R and Dong Z: hMSH2 recruits ATR to DNA damage sites for activation during DNA damage-induced apoptosis. J Biol Chem 286: 10411-10428, 2011.

18. Kolodner RD and Marsischky GT: Eukaryotic DNA mismatch repair. Curr Opin Genet Dev 9: 89-96, 1999.

19. Modrich P and Lahue R: Mismatch repair in replication fidelity, genetic recombination, and cancer biology. Annu Rev Biochem 65: 101-133, 1996.

20. Clark AB, Valle F, Drotschmann K, Gary RK and Kunkel TA: Functional interaction of proliferating cell nuclear antigen with MSH2-MSH6 and MSH2-MSH3 complexes. J Biol Chem 275: 36498-36501, 2000.

21. Flores-Rozas H, Clark D and Kolodner RD: Proliferation cell nuclear antigen and Msh2p-Msh6p interact to form an active mispair recognition complex. Nat Genet 26: 375-378, 2000.

22. Karpinich NO, Tafani M, Rothman RJ, Russo MA and Farber JL: The course of Etoposide-induced apoptosis from damage to DNA and p53 activation tomitochondrial release of cytochrome c. J Biol Chem 277: 16547-16552, 2002.

23. Rossi R, Lidonnici MR, Soza S, Biamonti G and Montecucco A The dispersal of replication proteins after Etoposide treatment requires the cooperation of Nbs1 with ataxia telangiectasis Rad3-related/Chk1 pathway. Cancer Res 66: 1675-1683, 2006.

24. Xia Y, Ongusaha P, Lee SW and Liou YC: Loss of Wip sensitizes cells to stress- and DNA damage-induced apoptosis. J Biol Chem 284: 17428-17437, 2009.

25. Oka S and Nakabeppu Y: DNA glycosylase encoded by MUTYH functions as a molecular switch for programmed cell death under oxidative stress to suppress tumorigenesis. Cancer Sci 102: 677-682, 2011. 